\title{
Kinematically Detected Halo Streams
}

To appear in "Tidal Streams in the Local Group and Beyond”, Astrophysics and Space

Science Library, Volume 420. Springer International Publishing Switzerland, 2016

Martin C. Smith

\begin{abstract}
Clues to the origins and evolution of our Galaxy can be found in the kinematics of stars around us. Remnants of accreted satellite galaxies produce overdensities in velocity-space, which can remain coherent for much longer than spatial over-densities. This chapter reviews a number of studies that have hunted for these accretion relics, both in the nearby solar-neighbourhood and the more-distant stellar halo. Many observational surveys have driven this field forwards, from early work with the Hipparcos mission, to contemporary surveys like RAVE \& SDSS. This active field continues to flourish, providing many new discoveries, and will be revolutionised as the Gaia mission delivers precise proper motions for a billion stars in our Galaxy.
\end{abstract}

\section{Introduction to kinematic streams}

At first glance the kinematics of disk stars in the solar neighbourhood might appear to be a smooth distribution, but upon closer inspection one can uncover a wealth of structure. The fact that this velocity distribution is clumpy has been known for over a century. The German astronomer J.H. von Mädler, while carrying out observations to measure the Sun's motion, noticed clumping in the distribution of proper motions (Mädler, 1846). This collection of stars moving with the same velocity, what we now refer to as a "moving group", consists of members of the Pleiades open cluster, including stars several degrees from the centre of the cluster. This work was build on by Proctor (1869), who found a further two moving groups - Hyades and Sirius.

The dissection of the local stellar velocity distribution has told us a great deal about star formation and Galactic structure. Figure 1 presents a contemporary analysis of the velocity distribution, showing that it is rich in substructure. These mov-

Key Laboratory for Research in Galaxies and Cosmology, Shanghai Astronomical Observatory, Chinese Academy of Sciences, 80 Nandan Road, Shanghai 200030, China; email:msmith@shao.ac.cn 
ing groups can be attributed to young open clusters which have not yet dispersed, or could be due to dynamical effects such as stars trapped at resonance with the Galactic bar or spiral arms (e.g. Dehnen \& Binney, 1998; Bovy \& Hogg, 2010; Sellwood, 2010; McMillan, 2011). However, neither of these will be explored in this chapter; interested readers are suggested to see Antoja et al. (2010) for a detailed and comprehensive review on the subject of moving groups. Instead we will here focus primarily on a third mechanism for creating moving groups, namely the accretion of extra-galactic systems.

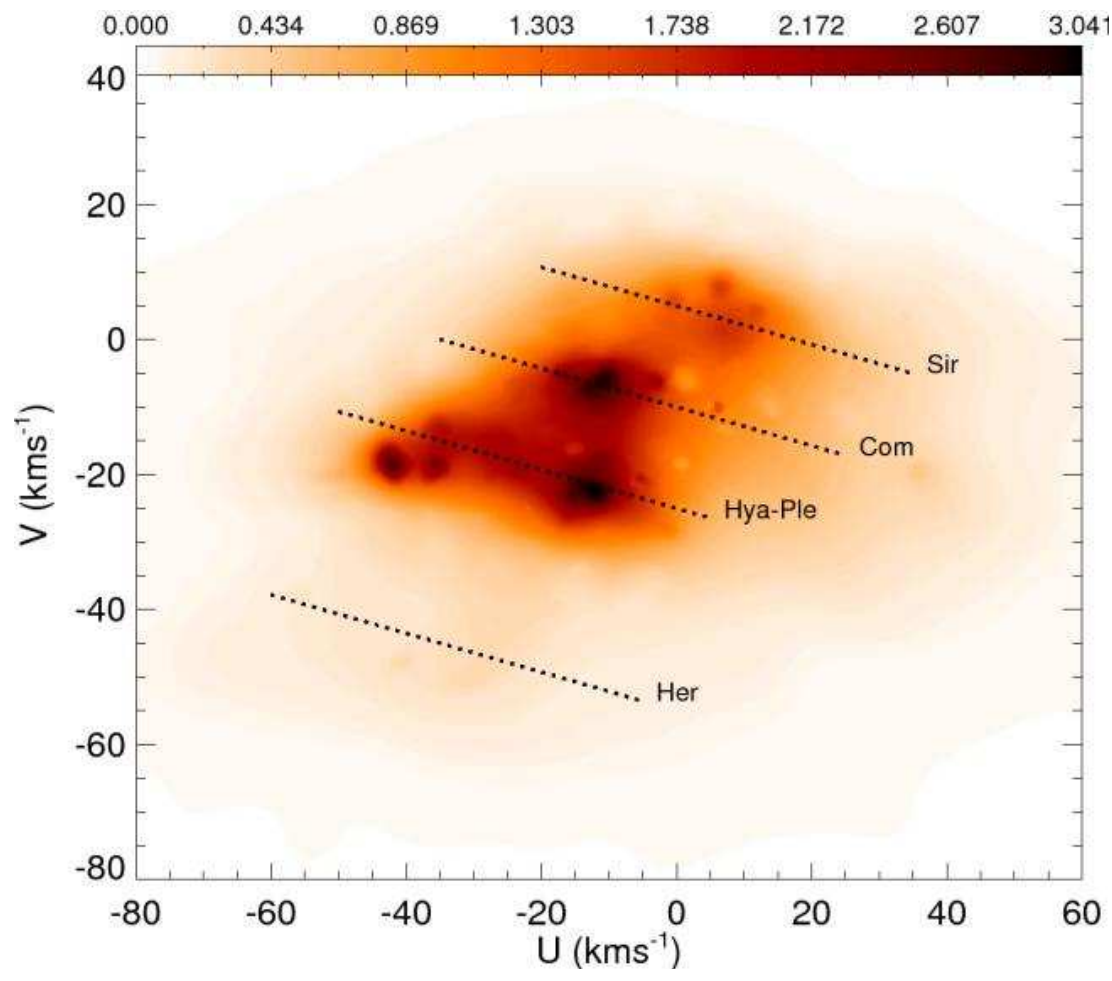

Fig. 1 The local stellar velocity distribution from a compendium of observational datasets. The two velocity components correspond to the Cartesian in-plane velocities, with $U$ increasing in the direction of the Galactic centre and V in the direction of the Sun's rotation. The approximate locations of four moving groups are denoted by the dotted lines. Figure taken from Antoja et al. (2010).

As can be seen from the other chapters in this volume, we know of many tidal streams in the stellar halo of the Milky Way. However, more diffuse streams, which are created if the progenitor is less massive, is on a highly eccentric orbit, or was tidally disrupted in the distant past, are harder to identify as spatial over-densities on the sky. They can, however, be discovered by exploring higher-dimensional space; 
a stream can that has dispersed in configuration space, may still remain coherent in phase space. In this chapter the many successful efforts to find fainter and more ancient accretion events will be reviewed, starting with the solar neighbourhood (Section 2) and moving out to structures in the more distant halo (Section 3).

\section{Local kinematic streams}

\subsection{The Helmi stream}

In the 150 years since Mädler made his initial discovery, many other studies have identified kinematic substructures. Some of these, such as the Arcturus or Kapteyn group, have been proposed to be extra-galactic in origin (we will return to these later). However, the first indisputable accretion remnant was found by Helmi et al. in their seminal 1999 paper (Helmi et al., 1999), which we will now discuss.

The first step to identifying clumping in velocity space is, by definition, obtaining a sample of accurate velocities. Large surveys have been crucial in propelling many fields in astronomy, from Kapteyn's visionary international survey of the early 20th century (Kapteyn, 1906; Kinman, 2000; van der Kruit, 2015), to the Sloan Digital Sky Survey in the early 21st (York et al., 2000); for a review of many significant results from such surveys in the field of Milky Way science, see Ivezić et al. (2012). One of the most important surveys in the past 20 years in the field of stellar kinematics was the Hipparcos mission. This was a European-led satellite mission, launched in 1989 and operated until 1993, which measured the position of stars to unprecedented accuracy. This enabled a catalogue of proper motions and parallaxes to be constructed for over 100,000 stars (ESA, 1997), although the complex nature of this task meant that the final definitive catalogue was only published in 2007 (van Leeuwen, 2007a,b).

Possessing such accurate proper motion data for large numbers of stars enables statistically meaningful analyses to be done of rare types of stars. For arguably the first time researchers were able to construct significant samples of stars from the halo, which remember constitutes only around 0.5 per cent of the stellar mass in the solar neighbourhood (Jurić et al., 2008). With this sample, one could now begin to search for kinematic over-densities.

The groundwork for Helmi's discovery was laid in the 1999 paper entitled "Building up the stellar halo of the Galaxy" (Helmi \& White, 1999). This paper used a combination of numerical simulations and analytic analysis to investigate the disruption of satellite galaxies as they are accreted into the Milky Way, paying particular attention to the consequences for the local stellar halo. By analysing the disruption of the satellite using action-angle variables, they studied how the tidal stream evolved over $10 \mathrm{Gyr}$, and showed that the system became phase mixed; at a particular location (for example in the solar neighbourhood) one might detect multiple kinematic over-densities from the same progenitor. This is because one (large) 
progenitor can produce a tidal stream that wraps multiple times around the Milky Way, and can produce numerous separate clumps in phase space. A simple calculation shows that if the local stellar halo is entirely made up of debris from 10-100 accreted satellites (of luminosity $1 \mathrm{e} 7$ or $1 \mathrm{e} 8 \mathrm{~L} \odot$ ) then one would expect to find a few hundred separate kinematic streams in the solar neighbourhood, although the individual stars in these streams would not necessarily be clumped in density.

Given the high quality data that was becoming available at that time, namely the aforementioned Hipparcos catalogue, Helmi et al. (1999) were able to construct a catalogue of 97 metal-poor red giants and RR Lyrae stars within $1 \mathrm{kpc}$ of the Sun (using data compiled by Beers \& Sommer-Larsen (1995) and Chiba \& Yoshii (1998) With distances estimated from photometry (to $\sim 20$ per cent), radial velocities from spectroscopy (to $\sim 10 \mathrm{~km} / \mathrm{s}$ ) and proper motions mainly from Hipparcos (to a few mas/yr), then were then able to analyse the phase-space distribution of this halo sample.

One then needs to determine the optimal space (combinations of $\mathrm{x}, \mathrm{y}, \mathrm{z}, \mathrm{v}_{\mathrm{x}}, \mathrm{v}_{\mathrm{y}}$, and $v_{\mathrm{Z}}$ ) in which to identify accretion debris. To do this one normally identifies integrals of motion, i.e. quantities that are conserved (or approximately conserved) along an orbit (see section 3.1.1 et seq of Binney \& Tremaine, 2008); stream stars are thus clumped in these quantities. For example, in a spherical potential we know that the total energy and the three components of angular momentum are all conserved along an orbit, leading to four integrals of motion. However, the Milky Way is not a spherical system, particular in the inner regions where the contribution of the disk to the total potential is important. Fortunately it is close to being axi-symmetric (since we do not need to worry about the Galactic bar, which is restricted to the innermost few kpc). For an axi-symmetric system, there are two integrals of motion: the energy, and the component of the angular momentum parallel to symmetry axis $\left(\mathrm{L}_{\mathrm{z}}\right)$. It can also be shown that the total angular momentum, although not precisely conserved, varies only slightly for modest amounts of flattening and, importantly, shows no long-term evolution.

There are small complications to this picture. First, the stars of a satellite galaxy do not all follow the same precise orbit, due to the internal velocity dispersion of the system. This means that stars will have a finite volume in this space defined by the integrals of motion. Second, the potential of the Milky Way will undoubtedly be varying over the time-scales under discussion, for example from the build-up of the disk as gas settles into the equatorial plane of the Galaxy. This leads to an oft-used term in this field - adiabatic invariants (see section 3.6 of Binney \& Tremaine, 2008). These are quantities that are constant for slowly-evolving potentials, i.e. potentials that vary on time-scales longer than typical orbital periods.

For their analysis, Helmi et al. (1999) chose to use the 2-dimensional space of $\mathrm{L}_{\mathrm{z}}$ and $\mathrm{L}_{\perp}=\sqrt{\mathrm{L}_{\mathrm{x}}^{2}+\mathrm{L}_{\mathrm{y}}^{2}}$. They did this as the former is conserved for an axi-symmetric potential and, since $\mathrm{L}^{2}=\mathrm{L}_{\perp}^{2}+\mathrm{L}_{\mathrm{z}}^{2}$ and both $\mathrm{L}$ and $\mathrm{L}_{\mathrm{z}}$ are approximately constant for stars in a particular tidal stream, the latter is not expected to vary significantly. These two quantities were selected because they are trivial to determine for their sample, unlike the energy which, although conserved, requires one to assume a model for 
gravitational potential. The distribution of $\mathrm{L}_{\mathrm{z}}$ and $\mathrm{L}_{\perp}$ is shown in Fig 2. For a local sample of stars $\mathrm{L}_{\mathrm{z}} \approx \mathrm{R}_{\odot} \cdot \mathrm{v}_{\phi}$, where $\mathrm{v}_{\phi}$ is the azimuthal component of the velocity. Since the stellar halo shows no significant signs of rotation (e.g. Smith et al., 2009b), one would expect that the distribution should be symmetric about $\mathrm{L}_{\mathrm{z}}=0$; clearly this is not the case at high $\mathrm{L}_{\perp}$, where there is a significant cluster of stars in the region $\left(\mathrm{L}_{\mathrm{z}}, \mathrm{L}_{\perp}\right)=(1000,2000) \mathrm{kpc} . \mathrm{km} / \mathrm{s}$. The probability of such a clump of 7 stars occurring by chance is estimated to be less than 1 per cent. Although their original halo sample contained only 97 stars, they augmented it with more distant and more metal-rich stars, obtaining a total of 12 stars that belong to this overdensity.

They also investigated the velocity distribution of this stars from this system (top panels of Fig. 2; see also Figure 1 of Chapter 1 which shows a subset of the sample in a slightly different coordinate system). Note that in Helmi's coordinate system, positive $L_{z}$ implies $v_{\phi}$ is in the same direction as Galactic rotation, so these stars have azimuthal velocities similar to the Sun, but with very high vertical velocities (as can be seen from the top panels of Fig. $2, \mathrm{v}_{\mathrm{Z}}$ is around $200 \mathrm{~km} / \mathrm{s}$ ). It is also interesting to see that $\mathrm{v}_{\mathrm{Z}}$ is split into two separate clumps - one moving downward and one upward. This is most-likely a manifestation of the aforementioned phase-mixing, where one progenitor can produce multiple streams in phase-space. So although there are two streams, these stars are all from the same accretion event. Helmi and collaborators constructed an N-body simulation of this system, which provides a very good representation of the observed velocity distribution (Fig. 2). This shows how the progenitor can be almost completely disrupted in configuration space, yet remain coherent in velocity space. The two separate clumps in $\mathrm{v}_{\mathrm{z}}$ are reproduced, as is the spread in $v_{R}$. The simulation also allows them to estimate the properties of the progenitor, which they concluded was likely to be similar in size to the Fornax dwarf spheroidal.

Subsequent studies have confirmed the Helmi stream and increased the number of member stars, though somewhat reducing the fraction of the halo it comprises (for example Chiba \& Beers (2000); Re Fiorentin et al. (2005); Dettbarn et al. (2007); Kepley et al. (2007) Later surveys containing fainter stars have enabled us to identify halo subdwarfs in this system (Klement et al., 2009; Smith et al., 2009b), bringing the total number of members to over 30 out to distances of a few kpc. The reported fraction of local stellar halo made of Helmi stream debris varies from reference to reference, probably due to differences in search techniques, but is most likely around 5 per cent. The chemistry of 12 of these stars has been investigated by Roederer et al. (2010). They find a spread in $[\mathrm{Fe} / \mathrm{H}]$ from -3.4 to -1.5 , with detailed abundances similar to that of the general halo population, concluding that star formation in the progenitor was truncated before the products of Type Ia supernovae or AGB stars enriched the inter-stellar medium.

The Kepley reference also introduces a novel way to estimate the age of accretion. They utilise the fact that once the progenitor has become completely phasemixed, the total number of stars in the positive and negative $\mathrm{v}_{\mathrm{z}}$ clumps would be the same. The observed fraction with positive $\mathrm{v}_{\mathrm{z}}$ is actually 28 per cent, which means it is not entirely mixed. By analysing an N-body simulation of the accretion (similar to the right-panel of Fig. 2), they can tentatively say that it was likely to have been 

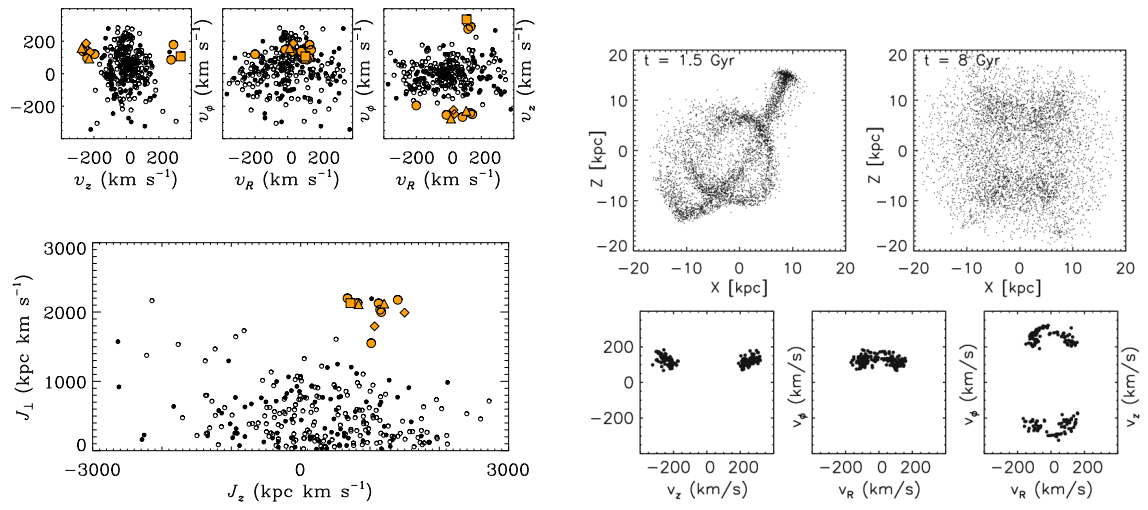

Fig. 2 The discovery of kinematic substructure in the solar neighbourhood, from Helmi et al. (1999). The left panel shows the observational discovery, which was based on an analysis of stars from the Hipparcos mission. The lower panel shows the distribution in angular momentum space (in their notation $\mathrm{J}=\mathrm{L}$ ), while the upper panel shows the kinematics. The right panel shows an $\mathrm{N}$-body model of the stream, which is almost entirely phase mixed after $8 \mathrm{Gyr}$. This panel was taken from Helmi (2008).

accreted between 6 and 9 Gyr ago, i.e. not too recently (otherwise the ratio would be more lopsided) and not too long ago (otherwise the ratio would be closer to unity).

\subsection{Other early discoveries}

As pointed out in the introduction, moving groups have been studied for many years, but definitively determining their origins is not easy. As a consequence, finding halo streams in the solar neighbourhood can be a precarious venture. Over-densities, as their name suggests, can only be identified as an excess on top of a background population. However, in this case the background population in question (the Milky Way disk) is far from a simple homogeneous population; lumps and gradients can often masquerade as coherent structures and so great care has to be taken in their classification. This problem has afflicted many potential stream discoveries and, as can be seen in this chapter, ambiguities still remain for a number of these.

Various streams, such as Hyades-Pleiades, Hercules and Arcturus, are believed to be Milky Way stars brought together by resonances in the disk (as mentioned in the introduction to this chapter). Originally identified by Eggen (see his 1996 review), the broad metallicity or age spread of these three streams argues against disruption of a disk star cluster as the origin, and abundance patterns similar to disk stars disfavours the extragalactic scenario, hence the conclusion of a dynamical origin (e.g. Famaey et al., 2008; Bovy \& Hogg, 2010; Fuchs \& Dettbarn, 2011). Some of these are more controversial than others; the Arcturus stream has generated much debate (e.g. Navarro et al., 2004), but more-recent analyses have shown that the narrow ve- 
locity dispersion (Bovy et al., 2009) and chemical inhomogeneity (Williams et al., 2009; Bensby et al., 2014) favor a dynamical origin. Making definitive claims about the origins of such systems is not easy.

Another stream that has been studied extensively is the Kapteyn moving group, again named by Eggen. The name comes from the fact that the velocities of this group are similar to that of Kapteyn's star, which is a well-known very high proper motion star only $4 \mathrm{pc}$ away from the Sun. This group is on a mildly retro-grade orbit, moving at around $-50 \mathrm{~km} / \mathrm{s}$.

Quite early it was speculated that this retro-grade group might be related to the globular cluster Omega Cen (e.g. Eggen, 1978), which is on a similar orbit. This cluster is interesting as it appears to possess a broad spread in both chemistry and age, indicating that it could be the core of a disrupted dwarf galaxy (Lee et al., 1999). This might suggest that this retrograde moving group is in fact the stripped remnants of the galaxy. A number of works have pursued this hypothesis (see Majewski et al. 2012 for a nice overview of the current situation), including a detailed spectroscopic analysis of 16 stars by Wylie-de Boer et al. (2010). This latter reference confirms the similarity in chemistry between the cluster and the moving group, but provocatively questions whether the group may in fact be material stripped from the host galaxy of Omega Cen as it was disrupted. The reason is that one would need a significant amount of dynamical friction to bring the system onto its current orbit, something which would not be possible for an object as small as Omega Cen. However, the authors acknowledge that their hypothesis is currently unproven; if they are correct that Omega Cen and the Kapteyn moving group do not originate in the same dwarf galaxy progenitor, then why the close similarity in chemistry and why the age spread in the cluster? Clearly more work needs to be done to address this question and fully understand the connection, especially given that a large fraction of the inner halo could belong to this moving group (see, for example, Majewski et al. 2012; Fig. 3) and hence it could have played a significant role in the early development of our galaxy.

\subsection{Streams in the Geneva-Copenhagen survey}

\subsubsection{Pieces of the puzzle}

Although Hipparcos was (and still is) clearly a hugely important resource for studying the kinematics of stars in the solar neighbourhood, it was hampered by a lack of radial velocities. What was needed was a systematic survey of radial velocities, providing a sample with a well-defined selection function. The Geneva-Copenhagen survey (Nordström et al., 2004; Holmberg et al., 2007, 2009; Casagrande et al., 2011) produced radial velocities and metallicities for 13,000 nearby stars. Combining these with proper motions and (for the majority of stars) distances from the Hipparcos mission, resulted in a complete sample of F- and G-type dwarfs within $40 \mathrm{pc}$ and a larger, magnitude-limited sample to around $200 \mathrm{pc}$. Although this is a 


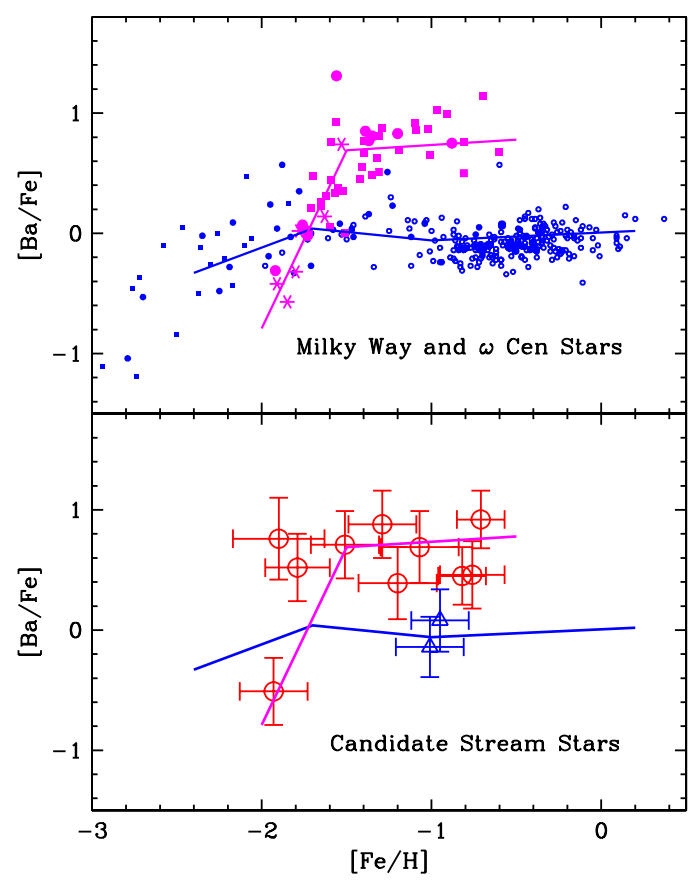

Fig. 3 Top panel: The distribution of $[\mathrm{Ba} / \mathrm{Fe}]$ vs $[\mathrm{Fe} / \mathrm{H}]$ for Milky Way stars (blue points) and a characteristic locus (blue line), overlaid with the same for $\omega$ Cen stars (magenta points and line), where data for both are taken from the literature. Bottom panel: the distribution of barium abundances for 10 stars in the retrograde kinematic stream discussed in Majewski et al. (2012; red), plus two retrograde stars not in the stream (blue). This retrograde stream is similar to the Kapteyn stream and is potentially associated to Omega Cen, a hypothesis which is supported by the close similarity in chemistry between the cluster stars in the top panel and the stream stars in the bottom. Taken from Majewski et al. (2012).

relatively local sample compared to the one analysed in Helmi's 1999 study, and contains only a handful of halo stars, the accurate $6 \mathrm{D}$ phase-space information allows a detailed analysis of the orbital properties of the stars.

In addition to the Helmi stream, which is the most prominent in the local stellar halo, a number of other candidate streams have been found. Helmi continued her analysis using this Geneva-Copenhagen survey (Helmi et al., 2006), choosing a different space to search for accretion debris than in their 1999 study. This time they chose three parameters, L_z (which, as mentioned above, will be conserved in an axi-symmetric system) and the peri- and apo-centre distances (the minimum and maximum distance that the star's orbit comes to the Galactic centre). We will refer to this as the APL space (Fig. 4). Using simulations they showed that disrupted satellites remain reasonably localised in this space, with the extent depending on the initial size of the satellite. When projected into the 2D space of apo- versus peri-centre distance, debris remain contained within a band of constant eccentricity, implying that the stars approximately retain the eccentricity of their progenitor's or- 
bit. Moreover in this space one can see the different kinematic streams forming as the satellite becomes phase mixed, although the accuracy required to determine this is not feasible with current datasets.

One clear drawback of this method is that it relies on a knowledge of the gravitational potential in order to calculate apo- and peri-centre distances. However, as the sample under investigation is such a small volume, the potential is approximately constant and hence the orbital parameters are determined by the kinematics, rather than their location. This means that although the apo- and peri-centre distances may be slightly off due to an incorrectly chosen potential, this will result in a systematic shift in this space and will not work to smear out any coherent features.

By comparing the density of stars in this 3D space to Monte Carlo realisations of a smooth model, they were able to show that their distribution is significantly more structured than what one would expect from a smooth model. They went on to identify a group of stars with eccentricities around 0.4 . This value is clearly inconsistent with the thin disk, although thick disk stars may posses such high eccentricities. Folding in metallicities appears to show that this group can be divided into three separate accretion events, with different trends in $v_{z}$ and age.

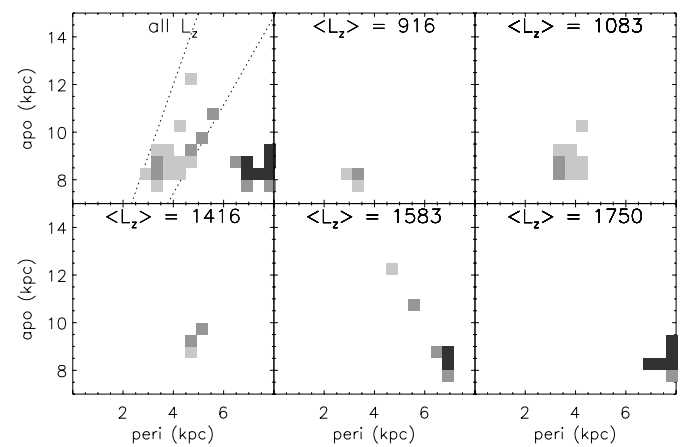

Fig. 4 Distribution of overdense cells in the apo-centre/peri-centre/angular-momentum (APL) space. Each panel shows the distribution of stars in the space of apo- and peri-centre (for different cuts in the L_z angular momentum). Darker colours correspond to higher statistical significance of an excess compared to the smooth background. Notice the group of stars confined within the dotted lines (corresponding to eccentricities between 0.3 and 0.5). As L $\_$z increases, the orbits move outwards in radii. Taken from Helmi et al. (2006).

The process of determining exactly how many distinct groups are contained in such a discovery is complex and open to interpretation. High-resolution follow-up studies have analysed the elemental abundance patterns of these stars and suggested that there may actually be only two distinct groups (Stonkutè et al., 2012, 2013; Ženovienė et al., 2014); the common abundance patterns suggests that Groups 2 \& 3 from the original Helmi et al. (2006) paper may come from the same progenitor. Tentative ages for these stars show two populations, a relatively metal-rich one of around $8 \mathrm{Gyr}$ and a more metal-poor one of around $12 \mathrm{Gyr}$, but they acknowledge 
that more work needs to be done as ages are notoriously difficult for main-sequence stars. They conclude by speculating that the similarity between the chemical composition of stars in these two kinematic groups and in the Milky Way's thick disk suggests that the progenitor of this system may be related to the formation of the thick disk.

The original authors revisited these streams in Helmi et al. (2014), obtaining high-resolution for even more stars in this eccentricity range (0.3-0.5). Although they no longer focus on the division proposed in their original paper, they show that the properties of stars in this eccentricity range are not homogeneous, with an apparent division around $[\mathrm{Fe} / \mathrm{H}]=-0.4$ dex.

\subsubsection{Arifyanto \& Fuchs}

In the same year as the Helmi et al. were mining the Geneva-Copenhagen survey, a group in Germany Arifyanto \& Fuchs (2006) were searching for accretion debris in a sample of 742 stars based on a catalogue from Carney et al. (1994). This resulted in the detection of a number of streams, but none are believed to be due to accretion; three had been previously identified (Hyades-Pleiades, Hercules and Arcturus; discussed above) and there was a new discovery, again not conclusively extragalactic in origin (Ramya et al., 2012).

However, despite not identifying any bone-fide accretion remnants, this paper warrants discussion here for its approach to identify nearby streams. Using a Keplerian approximation for orbits developed by Dekker (1976), they determine proxies for the total angular momentum and eccentricity, along with a third parameter corresponding to the inclination of the orbit (see Klement 2010 for a review of this method). They argue that this three dimensional space is ideal for dissecting velocity space, as evidenced by their success at finding the above disk streams. This method is simple to apply and unlike the previous APL approach does not require orbits to be calculated, but it is not perfect as it relies on the aforementioned Keplerian approximation that only holds for spherical potentials. Despite these drawbacks it has proved a popular approach that has been used by numerous authors, mainly by employing the proxy for the eccentricity $\left(\sqrt{U^{2}+2 V^{2}}\right)$. Although the approximations behind this proxy break down for high eccentricities, overdensities should remain coherent, and so the simplicity of this approach has resulted in its widespread use.

Another early paper to adopt this technique was Dettbarn et al. (2007), who analysed the sample of Beers et al. (2000) and found a number of candidate halo streams, including existing groups (such as the Helmi stream) and some new ones (named, $\mathrm{S}_{1}, \mathrm{~S}_{2}$, and $\mathrm{S}_{3}$ ). 


\subsection{The modern era}

\subsubsection{The RAVE survey}

It should be evident by now that progress in understanding the formation and evolution of our galaxy rests on large surveys. We will now discuss two influential surveys in this field from the past decade: the RAdial Velocity Experiment (RAVE) and the Sloan Digital Sky Survey (SDSS). Both of these have led to numerous discoveries, as can be seen from this and many other chapters in this volume.

Taking a kinematic census of large populations of the Milky Way requires both photometric and spectroscopic surveys. Many proper motion catalogues have been assembled, often by combining various existing ground-based astrometric surveys (e.g. the Hipparcos catalogue, UCAC4, PPMXL, etc, etc). Spectroscopic surveys are in some sense rarer as it requires significant telescope time to amass spectra for hundreds of thousands of stars. One of the largest such surveys is the RAVE survey (Steinmetz et al., 2006), which began in 2003 on the 1.2m UK Schmidt Telescope at the Anglo-Australian Observatory. Despite not being particularly new (it was around 30 years old when the survey started) or having a large aperture, it had a number of important assets: the field of view was very large (6 degree diameter field); it had relatively high multiplexing capabilities (150 fibers per plate); and, crucially, for most of the survey it had dedicated use of the telescope. The combination of these factors allowed RAVE to efficiently produce large catalogues of radial velocities and abundances, resulting in a final catalogue of around 500,000 stars in the Southern hemisphere with magnitude range $9<\mathrm{I}<12$ (the fourth public data release is presented in Kordopatis et al., 2013). By concentrating on a narrow window around the calcium triplet region (8410-8795 A) with reasonable resolution $(\mathrm{R} \sim 7500)$, the radial velocity accuracy is excellent, at around a couple of km/s (see Fig. 29 of Kordopatis et al., 2013). Furthermore, stellar parameters can be derived at a level of around a few tenths of a dex for $\log (\mathrm{g})$ and metallicity (see Table 2 of Kordopatis et al., 2013). . For the higher signal-to-noise spectra it is even possible to estimate abundances for a number of individual elements, such as $\mathrm{Mg}, \mathrm{Al}, \mathrm{Si}, \mathrm{Ca}, \mathrm{Ti}, \mathrm{Fe}$, and $\mathrm{Ni}$ (Boeche et al., 2011; Kordopatis et al., 2013). If we are interested in learning about the kinematics of stars, the final piece of information is distances, which can be estimated by combining the observed stellar parameters with stellar models. This was first undertaken by Breddels et al. (2010) and subsequently refined by Zwitter et al. (2010) and Binney et al. (2014b).

With all this information in hand, RAVE has proved to be a hugely important survey for understanding the kinematics of the solar-neighbourhood, for example Binney et al. (2014a). In terms of streams, the first attempt to investigate this was Seabroke et al. (2008). By looking at stars from a couple of surveys, including RAVE, they came to the conclusion that there were no massive streams (with hundreds of stars) present in the dataset. They contrasted this to the situation in the outer halo (see Section 3 of this chapter, as well as other chapters in this volume), where the system is clearly far from relaxed and contains a number of streams and substructures. The results here are in agreement with previous studies, for example 
the work of Gould (2003), which used a sample of 4,000 halo stars with proper motions to conclude that no more than 5 per cent of the local halo can be made up of a single stream. At first glance this may appear to conflict with some estimates for the density of the Helmi et al. (1999) stream discussed above, but one should note that their discovery is made up of two separate kinematic streams due to the extensive phase-mixing (Fig. 2).

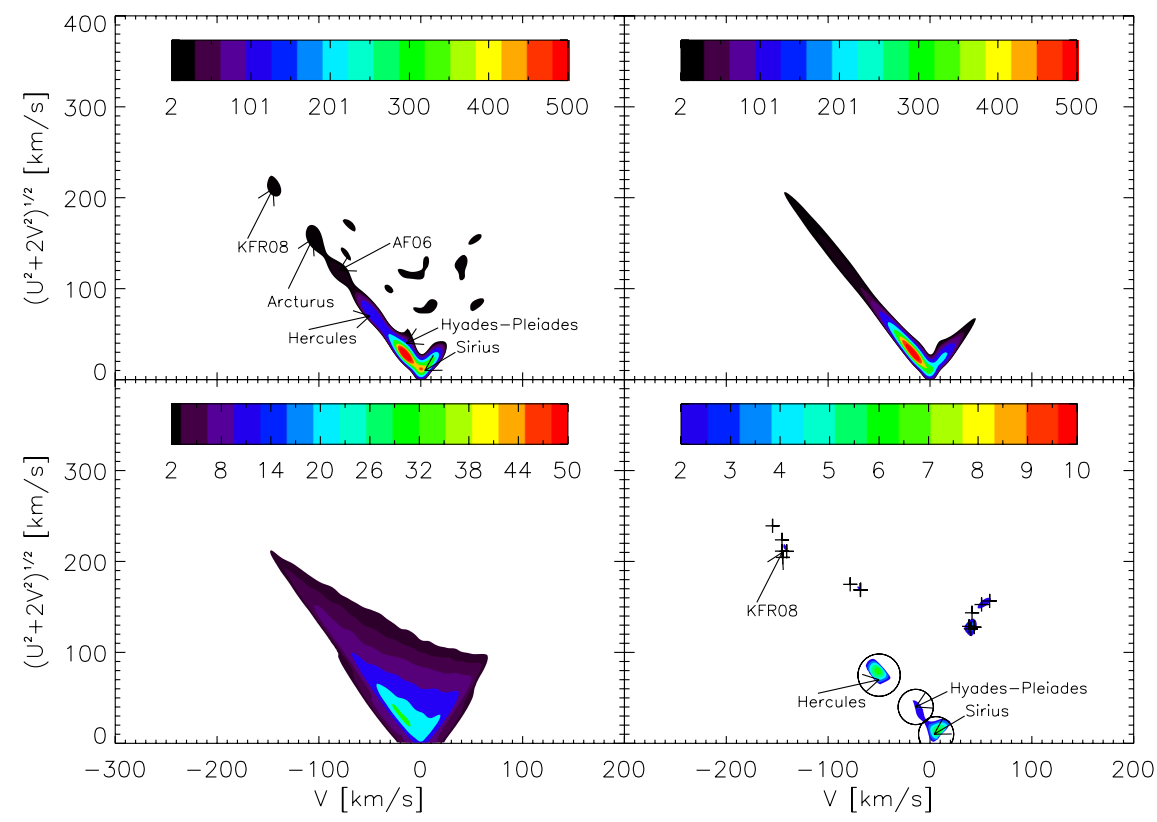

Fig. 5 Distribution of kinematic over-densities in RAVE, as presented by Klement et al. (2008). The upper-left panel shows the full distribution of the observed dataset, which can be compared to the expected distribution from a smooth model (upper-right) and its corresponding dispersion (lower-left). By subtracting the data from the model, normalised by the dispersion, one obtained the distribution of overdensities (lower-right). This reveals known substructures, such as Hercules, and new ones, such as KFR08. This figure is taken from the later analysis presented in Klement et al. (2011).

The first attempt to systematically search for small-scale clumping in velocity space in the RAVE survey was undertaken by Klement et al. (2008), who adopted the Keplerian approximation described in the previous section. One issue here is that 6D phase-space is required and, back in 2008, distances has not yet been estimated for RAVE stars. To overcome this issues they made the naive assumption that the sample would be dominated by main-sequence stars and applied a simple monotonic colour-magnitude relation suitable for dwarf stars. Due to the bright magnitudes that RAVE probes, this assumption is rather weak and it was subsequently found that around half of the stars are likely to be giants or sub-giants (e.g. Binney et al., 2014b). They later rectified this issue (Klement et al., 2011), basically finding 
that their results were unchanged. Despite this problem in their original paper, they were still able to recover a number of existing streams, calculating their significance by comparing to expectations from a smooth background model (Fig. 5). One new stream (dubbed "KFR08") was also detected at a level of 3-sigma. Despite being relatively metal-rich $(-1<[\mathrm{Fe} / \mathrm{H}]<0$ ) and on a pro-grade orbit (with v_phi of around $160 \mathrm{~km} / \mathrm{s}$ ), the high vertical velocity (vertical dispersion being around $100 \mathrm{~km} / \mathrm{s}$ ) indicates that this unlikely to be associated to the disk and is therefore believed to be the remnant of an accreted satellite. This was later analysed by Bobylev et al. (2010) in a sample constructed from Hipparcos stars with accurate trigonometric parallaxes (van Leeuwen, 2007a) and metallicities and ages (Holmberg et al., 2007, 2009); by identifying additional giant stars in this stream they were able to identify the main sequence turnoff and, through isochrone fitting, determining this is likely to be a very old stream (likely $13 \mathrm{Gyr}$ in age). We will return to this stream briefly in the following section, as it is was subsequently confirmed using an independent data set.

As discussed above, determining the origin of moving groups can be tricky due to the complex nature of the background in which they reside (i.e. the Milky Way disk). This difficulty is highlighted by another candidate stream identified by the RAVE survey, entitled the Aquarius Stream (Williams et al., 2011). Is was discovered through its radial velocity offset from the surrounding population (Fig. 6) a cluster of 15 stars localised on the sky and with very similar metallicities. This old stream was not connected to any other existing structures and they concluded that it was likely recently stripped material from a globular cluster or dwarf galaxy. However, despite being relatively close by (most of the stars are within a few kpc), uncertainties in the distances and proper motions mean that the clumping in angular momentum space is inconclusive.

At this point detailed abundances are required to definitely determine the origin of this stream. Two teams took up this task, one led by Wylie-de Boer et al. (2012) using the AAT telescope and one led by Casey et al. (2014) using the Magellan Clay telescope. Unfortunately, instead of clarifying the issue, these studies raised more questions than they answered. The first analysis (Wylie-de Boer et al., 2012) looked at six member stars, concluding that the exceptionally tight metallicity spread $(\sigma([\mathrm{Fe} / \mathrm{H}])=0.1 \mathrm{dex})$ and abundance ratios unambiguously show that the Aquarius stream is a disrupted globular cluster. The second analysis (Casey et al., 2014), on the other hand, looked at five stars and unambiguously found that this system cannot be a disrupted globular cluster, giving their paper the unequivocal title "The Aquarius comoving group is not a disrupted classical globular cluster". After finding a wide metallicity spread $(\sigma[\mathrm{Fe} / \mathrm{H}]=0.4 \mathrm{dex})$ that is inconsistent with a globular cluster and abundance ratios that are inconsistent with a dwarf galaxy, they conclude that the stars are indistinguishable from the Milky Way field population. The coherence in their kinematics may be a result of the perturbation of the disk when a satellite fell in, but this is just conjecture and their main conclusion is that the chemistry of these stars clearly show that they are not the accreted remnants of either a globular cluster or dwarf galaxy. The fact that any two studies disagree is not surprising. What IS surprising is that one cannot easily explain away 
this discrepancy on the grounds of small number statistics, since these two analyses have four stars in common! So the fact that very different conclusions have been reached is hard to reconcile, although the higher signal-to-noise of the Casey et al. study certainly works in their favour and they consequently argue that the Wylie-de Boer et al. metallicities may be inaccurate. To conclude, the nature of the Aquarius stream/group is still open for debate.
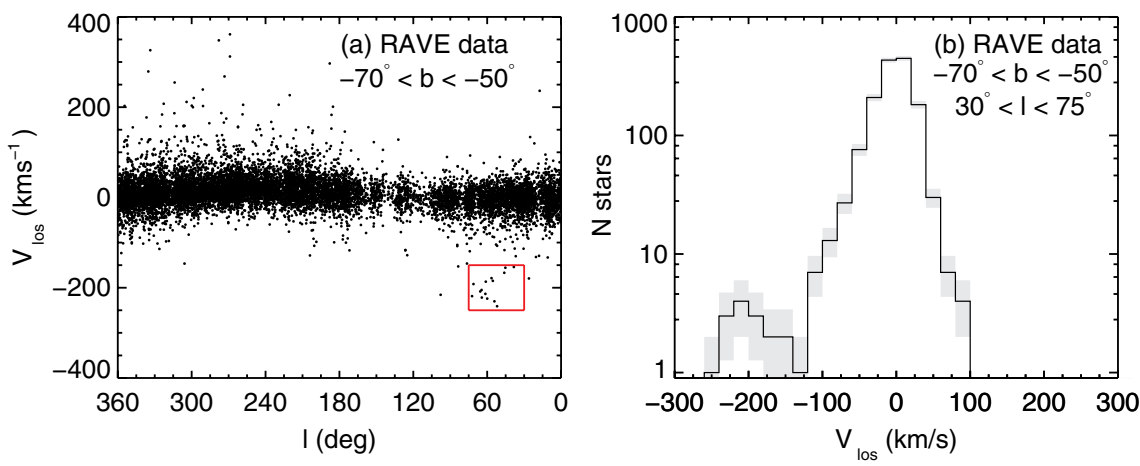

Fig. 6 These plots, from Williams et al. (2011), show the disputed Aquarius stream/group. Despite the strong signature in kinematics (which we see localised at $\mathrm{v}_{\text {los }} \sim-200 \mathrm{~km} / \mathrm{s}$ ), it is still argued whether this is the remnant of a disrupted globular cluster (Wylie-de Boer et al., 2012) or just a perturbation in the disk (Casey et al., 2014).

\subsubsection{The SDSS survey}

In terms of studies of the Milky Way, another major survey in the past decade has been the Sloan Digital Sky Survey (SDSS). The photometric part of the survey has led to the discovery of a host of new streams and dwarf galaxies, including a new population ultra-faint dwarf galaxies (see Belokurov 2013 for a recent review). Furthermore it has spurred on many different analyses on the state of the disk both through its photometry and spectroscopy (for example, see review articles by Ivezić et al. 2012 and Smith et al. 2012).

The sub-project dedicated to stellar spectroscopy, called the Sloan Extension for Galactic Understanding and Exploration (SEGUE; Yanny et al. 2009), was included in SDSS-II and SDSS-III and collected spectra for nearly 350,000 stars. There were also a large number of stars targeted in the other sub-projects (over 300,000) and although they have a very complex selection function, these stars are still useful for studies of the Milky Way. These SDSS spectra were taken at a much lower resolution than RAVE (with $\mathrm{R} \approx 2000$ ) but with a much broader wavelength coverage (38009200 A). With this data the SDSS team were able to measure stellar parameters to a precision similar to that of RAVE, namely $\log (\mathrm{g})$ and $[\mathrm{Fe} / \mathrm{H}]$ to around 0.3 dex (Lee et al., 2008). Alpha elements have also been obtained (Lee et al., 2011), 
but of course these are harder to determine and, at the time of writing (i.e. Data Release 12), are yet to be officially released as part of the SDSS survey. Despite not being officially released, a number of authors have used these alpha-element abundances, for example measuring the abundance "knee" of the Sagittarius stream (de Boer et al., 2014). The spectroscopy has also led to a variety of papers on the Milky Way disk, most provocatively the series of papers led by Jo Bovy arguing that the thick disc is not a separate entity from the thin disk (Bovy et al., 2012). The SDSS survey is still ongoing, with the stellar spectroscopy now undertaken by the ambitious APOGEE project, which will be discussed later in Section 4.

The search for phase-space substructures in SDSS was undertaken by Klement et al. (2009) and Smith et al. (2009b), using complimentary datasets and techniques. Klement et al. took the seventh data release from SDSS and applied the same methods as used during their search for overdensities in the RAVE survey. Since the SDSS stars are much fainter than the RAVE stars, proper motions are generally less precise. Therefore in order to reduce the corresponding uncertainties in tangential velocities, they decided to focus on stars within $2 \mathrm{kpc}$ of the Sun. They were able to confirm their candidate overdensity from the RAVE survey (the KFR08 group, discussed above), further breaking this into two separate groups, one of which is likely to have a disk origin (R1) and one of which is likely accretion debris (R2). They also confirm one of the previously mentioned streams from Dettbarn et al. (2007), adding more members and arguing that this is actually smeared out in phase-space, resulting in multiple clumps in their analysis. As with most works, the Helmi stream was found with very strong significance. Finally, two additional candidate halo streams were identified ( $\mathrm{C} 1 \& \mathrm{C} 3)$.

At the same time as Klement et al. (2009) were working on their paper, Smith et al. (2009b) were working on an independent study using SDSS data. Their approach was complimentary in that it was able to probe much greater distances thanks to precise proper motions from Bramich et al. (2008). These proper motions were constructed using the 250 sq. deg. Stripe 82 region of SDSS, which was repeatedly monitored primarily for the purpose of detecting supernovae. However, the multiple epochs are also ideal for constructing proper motion catalogues. The fact that the proper motions come from a single survey avoids the problem of cross-matching data from separate telescopes with often very different conditions and imaging systems, significantly reducing the systematic errors. Despite the relatively short baseline (seven years), proper motions were measured to a few mas/yr precision, even at faint magnitudes. Koposov et al. (2013) updated the catalogue using improved techniques, reducing the systematic errors even further and obtaining a precision of around 2 mas/yr. The stunning precision enabled the authors to detect the proper motion of the Sagittarius stream, even at a distance of $30 \mathrm{kpc}$.

These accurate proper motions allowed Smith et al. to investigate halo stars out to $5 \mathrm{kpc}$, with accuracy around $30-50 \mathrm{~km} / \mathrm{s}$ for each component of the velocity. The halo stars were identified using a reduced proper motion diagram (Fig. 7). This technique, which uses the proper motion as a proxy for distance, is often used to separate (nearby) dwarfs from (distant) giants, but is a powerful technique to separate halo stars from disk stars, as halo stars are faster moving and hence have higher proper 
motions for a given distance. Although this selection is kinematically biased, it is easy to correct for this as the cut in reduced proper motion corresponds to a cut in tangential velocity; from a simple model one can calculate the detection efficiency for stars of a given velocity without accurate distances or luminosity functions. An additional factor which helps to separate out halo stars is that metal-poor stars are bluer, meaning that (for a given colour) metal-poor stars are intrinsically fainter than metal-rich ones by as much as a couple of magnitudes (see, for example, equation A2 of Ivezić et al. 2008; see also Bochanski et al. 2013). Once the halo stars were identified photometrically from the reduced proper motion diagram, they were cross-matched with SDSS spectroscopy to provide metallicities and velocities. Distances were estimated by combing spectroscopic metallicities with the photometric distance relation of Ivezić et al. (2008), amended slightly to include a minor correction as one approaches the turn-off region (see Appendix B of Smith et al. 2009b and the Appendix of Smith et al. 2012). Here also the Stripe 82 data were beneficial; the multiple epochs provide much improved photometric accuracy compared to the rest of the SDSS footprint (e.g. median (g-i) error was less than $10 \mathrm{mmag}$ ), enabling much more precise photometric distances.

This halo sample was exploited for a variety of studies, including measuring the tilt of the halo velocity ellipsoid out of the plane (Smith et al., 2009a) and investigating the global kinematics (Smith et al., 2009b). In the context of this chapter, the sample was also important for investigating halo substructures. By working in angular momentum space $\left(\mathrm{L}_{\mathrm{Z}}, \mathrm{L}_{\perp}\right)$, the sample shows clear evidence for the Helmi stream, for the first time detecting candidate members up to $5 \mathrm{kpc}$ away (see Fig. 7). New candidate substructures were also found, dubbed Sloan Kinematic Overdensities (SKOs). The first one of these (SKOa) is particularly interesting - detected as a weak overdensity of stars with high $\mathrm{L}_{\perp}$, it turned out that the feature coincides (in angular $\mathrm{L}_{\mathrm{z}}-\mathrm{L}_{\perp}$ space) with a number of globular clusters taken from Dinescu et al. (1999). Figure 10 of Smith et al. (2009b) shows how these four clusters (NGC 5466, NGC 6934, NGC 7089/M2 and NGC 6205/M13) lie apart from the main distribution, raising the intriguing possibility that SKOa may be either the remains of tidal debris from one of these clusters or alternatively the remnant of a larger galaxy which hosted a number of smaller systems (i.e. these clusters, possibly including the progenitor of SKOa). Such hypotheses are difficult to test, but a detailed abundance analysis will be able to determine if its chemistry is consistent with any of these four globular clusters.

The remaining two over-densities, (SKOb \& c) arose from a search for distant systems that are localised on the sky. The orientation of this field (it is a long, thin stripe measuring $2.5 \times 100 \mathrm{deg}$ on the sky) means that coherent streams are likely to "cut through" this narrow stripe, and so Smith et al. (2009b) sliced the field along its length and searched for clumping in angular momentum space. Of the two overdensities, the most prominent is SKOb. This has been confirmed using data from MMT (Smith et al., in prep; see Fig. 8), pinning down the distance to between 4 and $5 \mathrm{kpc}$. This structure is intriguing because, unlike most of the other halo moving groups mentioned in this Chapter, it appears to be coherent, i.e. more like a stream than a moving group. As it is close enough to measure proper motions, this means 

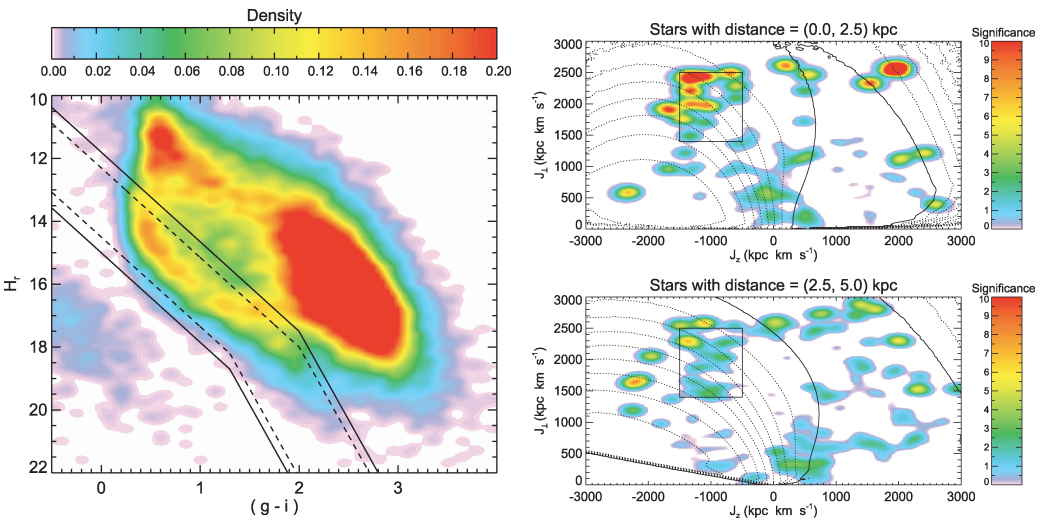

Fig. 7 Analysis of kinematic overdensities in SDSS subdwarfs. The left panel shows how the subdwarfs can be selected from a reduced proper motion diagram. The subdwarfs, which are located within the dotted lines, separate from the disc sequence owing to their fainter intrinsic magnitudes and greater velocities. The two right panels show the resulting distribution of subdwarfs in angular momentum space, after subtraction of a smooth halo model. Note the prominent Helmi stream, located around $\mathrm{J}_{\mathrm{Z}}=-1000$ and $\mathrm{J}_{\perp}=+2000 \mathrm{kpc} \mathrm{km} / \mathrm{s}$. The dotted lines in these panels denote the detection efficiency, which has to be considered as these stars are kinematically selected. Figure taken from Smith et al. (2009b).

that if we can trace an extension of this system across the sky it will become a $6 \mathrm{D}$ stream, which are very rare and important for modelling the halo (e.g. Koposov et al., 2010). As with SKOa (and, for that matter, the other overdensities found by Klement et al. in SDSS), detailed abundances would be useful to better understand the origins of this system. However, as SDSS photometric data extends to relatively faint magnitudes, high resolution follow-up studies are prohibitively expensive in terms of telescope time.

\section{Distant halo streams}

The above discussion of local halo streams focused on those found using threedimensional kinematics. However, once we move beyond the solar neighbourhood, uncertainties in proper motion prohibit the use of tangential velocities. Tangential errors scale linearly with distance; for example, a proper motion error of $1 \mathrm{mas} / \mathrm{yr}$ at $1 \mathrm{kpc}$ corresponds to a tangential error of $4.7 \mathrm{~km} / \mathrm{s}$, but at $10 \mathrm{kpc}$ this error will grow to $47 \mathrm{~km} / \mathrm{s}$. As a consequence, any detailed structure in phase-space is washed out. Fortunately radial velocity uncertainties do not scale with distance and so these can be used to probe large volumes of the halo, with the caveat that it is now harder to interpret any identified substructures, as two components of the phase-space are missing. Also working in our favour is that in the outer halo the mixing times are 


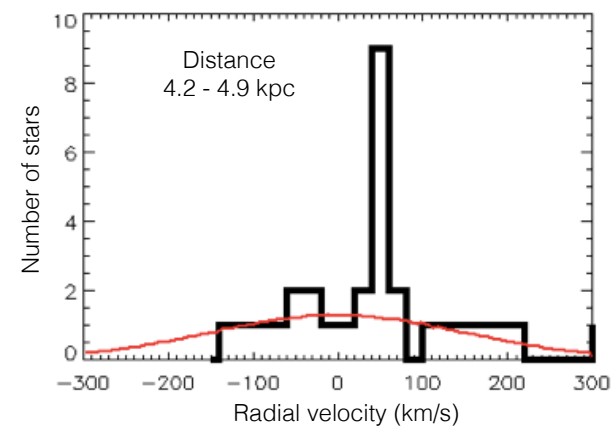

Fig. 8 Confirmation of the SKOb overdensity, which was initially discovered by Smith et al. (2009b). This figure shows the radial velocity distribution of stars with distances between 4.2 and $4.9 \mathrm{kpc}$, exhibiting a clear peak at $+60 \mathrm{~km} / \mathrm{s}$. The red curve denotes the expected distribution for the smooth halo.

much longer, which allows ancient structures to remain coherent in configuration space for many Gyr.

The most spectacular and important discovery of halo substructure through radial velocities is that of the Sagittarius dwarf galaxy. For many years observers had found dwarf galaxies around the Milky Way, beginning with the Magellanic Clouds, but one lay hidden behind the bulge of the Milky Way and was only discovered in 1994 (Ibata et al., 1994). This was uncovered serendipitously during a radial velocity survey of stars towards the Galactic bulge. When analysing the radial velocity distributions in certain fields, instead of the expected Gaussian distribution, a secondary peak was identified. This peak corresponded to the Sagittarius dwarf galaxy, whose systemic radial velocity is offset from the bulge by around $150 \mathrm{~km} / \mathrm{s}$. Subsequent works have revealed that this dwarf is in the process of being devoured by our Galaxy, with tidal streams being discovered encircling the entire Milky Way (see Chapter 2).

At higher latitudes other streams have been identified by their coherent radial velocities, including the Cetus polar stream (Newberg et al., 2009), the cold metalpoor stream of Harrigan et al. (2010) and the high-velocity stream of Frebel et al. (2013).

One of the most impressive studies of radial-velocity selected substructures in the halo was led by Kevin Schlaufman in the "ECHOS" series of papers (Schlaufman et al., 2009, 2011, 2012). Their approach was to take each SDSS/SEGUE spectroscopic plate and determine, using robust statistics, whether the radial velocity distribution of the main-sequence stars matches what one expects for a smooth halo. This was done using two statistical tests, as described in Section 3.2 of Schlaufman et al. (2009) and illustrated in Fig. 9. The first test compared the radial velocity 
histogram to a similar histogram (with the same number of radial velocity measurements) drawn from a smooth model halo. This realization of the smooth halo was repeatedly resampled in order to test the significance of any peaks in the observed distribution. As can be seen from the upper panel of Fig 9, the grey shaded region shows the 95 per cent confidence interval from these realisations of the smooth halo model; the fact that the observation (black histogram) and its error bar do not overlap the grey shaded region implies that this is a robust detection. The second test is based on the cumulative distribution of velocities, which retains more information than the previous approach (i.e. unlike the previous approach, it avoids any binning of the data). Again the observed distribution is compared to one drawn from a smooth model, but this time they compare the steepness of the cumulative distribution function. This is shown in the lower panel of Fig. 9, where one can see that the observed slope (given by the black curve) reaches into the high-significance regions (shown by the dark grey region).

Schlaufman et al. applied these techniques to observations of main-sequence halo stars out to $17.5 \mathrm{kpc}$ on 137 individual spectroscopic plates of SEGUE data. In these 137 lines of sight, they identified a total of 10 strong candidates (where the number of false positive detections is estimated to be less than 1) and a further 21 weaker candidates (estimated to have less than 3 false positives). A number of these are likely to be detections of existing substructures, such as the Monoceros stream, but 7 of the strong candidates are new detections. Note that this does not translate to 7 new independent halo substructures, as some streams could intersect multiple lines of sight, but it does show that the halo of the Milky Way is (at least in terms of its kinematics) lumpy, even in the inner halo where phase mixing should occur on relatively small time-scales. Schlaufman et al. quantify this "lumpiness," concluding that around 34 per cent of the inner halo is in the form of elements of cold halo substructures (ECHOS) and estimate that there could be as many as 1e3 individual kinematic groups in the entire inner halo.

The chemical composition of these ECHOS was investigated in the series' second paper (Schlaufman et al., 2011). They found that these ECHOS were more iron-rich and less alpha-enhanced than the smooth halo, concluding that the most-likely origin is that they were formed from the tidally disrupted debris of relatively massive dwarf galaxies $\left(\mathrm{M}_{\mathrm{tot}}>1 \mathrm{e} 9 \mathrm{M}_{\odot}\right)$. In the final paper of this series (Schlaufman et al., 2012) the authors investigated the spatial coherence in $[\mathrm{Fe} / \mathrm{H}]$ as a function of Galactocentric radius, concentrating only on main sequence turnoff stars from the kinematically smooth halo (namely SEGUE fields in which no ECHOS were detected). Although these fields are phase mixed and show no kinematic substructures, the chemistry of these stars can illuminate their origins. By studying the distributions of stars in the $[\mathrm{Fe} / \mathrm{H}]-[\alpha / \mathrm{Fe}]$ plane they found that the accreted halo becomes dominant beyond around $15 \mathrm{kpc}$ from the Galactic centre, arguing that at smaller radii the halo is probably formed from a combination of in-situ star formation and dissipative major mergers at high redshift.

As the careful work of Schlaufman et al. has shown, statistical studies are important if we are to dissect the halo of the Milky Way beyond the solar neighbourhood, especially when the sky coverage is not contiguous and the sampling of stars 


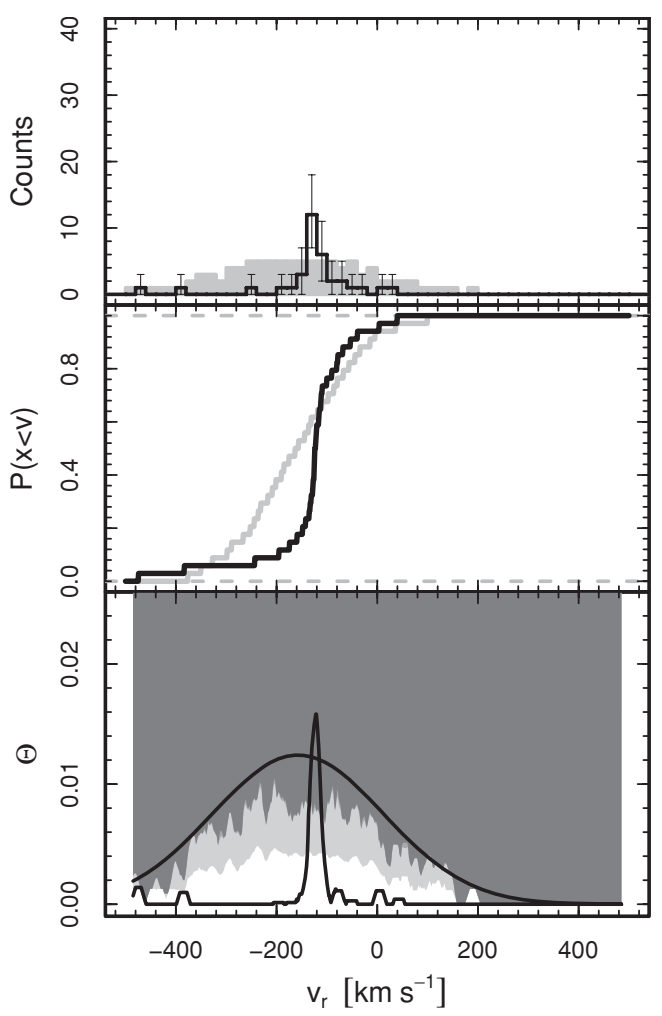

Fig. 9 Example of the search for an ECHOS from Schlaufman et al. (2009). The upper panel shows the radial velocity distribution (black histogram with error bars) and the 95 per cent confidence interval from the multiple realisations of the smooth halo model (grey shaded histogram). The middle panel shows the cumulative distribution function from the observed data (black) and mean from the realisations of the smooth halo model (grey). The lower panel shows their measure of the statistical significance Theta (black line), with the various significance levels denoted in light and dark grey. The wide Gaussian in this panel shows an envelope around the most significant region, which is included to remove any spurious detections due to small-scale fluctuations.

with spectra is sparse. Various tools have been developed, including the 4distance measure introduced by Starkenburg et al. (2009; see also the similar approach of Clewley \& Kinman 2006). This technique, which is based around the separation of pairs of stars in four dimensions (angular position on the sky, distance and radial velocity), has proved influential for subsequent works (e.g. Cooper et al., 2011; Xue et al., 2011) and will undoubtedly continue to be used on surveys of distant halo stars where proper motions are unavailable. 


\section{Future prospects}

Despite extensive progress in identifying kinematic streams, the field is far from exhausted. On the contrary, this decade is likely to see a resurgence in this field, leading to unprecedented insights into the formation of our Galaxy.

From the current generation of spectroscopic surveys, we can expect significant progress in the coming years. In terms of sheer volume of spectra, the Chinese LAMOST survey is unsurpassed (Deng et al., 2012). By gathering over a million spectra each year, this spectroscopic survey has great potential. Already one new candidate kinematic overdensity has been identified (Zhao et al., 2014) and various works are analysing substructure in the local velocity distribution, for example the work of Xia et al. (2015) which is utilising the extreme deconvolution technique (Bovy Jo et al., 2011).

If we think about the accreted galaxies which built up our stellar halo, they will of course have a range of masses and accretion times. Their chemical composition will therefore vary since the amount of enrichment that can take place depends on these factors (see, for example, Lee et al. 2015). As a consequence, a detailed dissection of the accretion history of our halo will require both kinematics and chemistry. By combining dark matter simulations with semi-analytic prescriptions for the star formation and chemistry, it is possible to make predictions for what we may be able to detect and how much we can infer about our Galaxy's accretion history from a given set of of kinematic and chemical abundance data (e.g. Johnston et al., 2008).

There are a number of spectroscopic surveys that operate at resolutions sufficient to carry out detailed chemical abundance analyses, for example the SDSS project APOGEE (Holtzman et al., 2015), the GALAH survey (Freeman, 2012), or the Gaia-ESO survey (Gilmore et al., 2012; Randich et al., 2013). These detailed abundances opes up the possibility of "chemical tagging," whereby abundance ratios are used to disentangle the different formation sites for groups of stars (Freeman \& Bland-Hawthorn, 2002). Clearly these additional dimensions will be extremely valuable when attempting to identify kinematic substructures in the local disk, where groups may overlap if one looks at only the 6D phase-space. Although this technique is ideally suited to finding moving groups in the disk, as mentioned above chemistry will allow us to classify halo streams and understand their origins - in effect carrying out the discovery and follow-up in one step.

As the Gaia satellite begins to deliver scientific return, there is no doubt that we are on the cusp of a true revolution in this field. This mission, which is led by the European Space Agency, is collecting high precision astrometry of a billion stars in our galaxy. All stars in the sky brighter than 20th magnitude will be observed, leading to exquisite proper motions and parallaxes. The precision is so great that it will be able to measure distances (through trigonometric parallax) to less than 1 per cent for ten million stars. In addition to the astrometry, Gaia will provide detailed photometric information (from spectrophotometry) including stellar parameters and, for stars brighter than around 17th magnitude, spectroscopic information including radial velocities. A description of the science capabilities can be found in de Bruijne (2012), although continually updated performance information can be found on the 
Gaia webpage. The final catalogue is expected in 2022, with interim releases before then.

Clearly such an unprecedented mapping of 6D phase space will open up an entirely new view of the local velocity distribution. While we wait for the first Gaia data to appear, many authors have attempted to estimate what we might be able to see. One example of this is Gómez et al. (2010), who modelled the Milky Way halo through the accretion of satellite galaxies, then convolved these with Gaia's observational errors. Fig. 10 shows what we may be able to detect in a solar neighbourhood realization; there are $1 \mathrm{e} 5$ stellar halo particles within this sphere of $4 \mathrm{kpc}$ radius, plus around 20,000 stellar disk particles. Upon applying a detection algorithm to identify substructure, they confirm 12 separate accretion events, corresponding to around 50 per cent of all disrupted satellites in this volume. For some of these detections the authors find that it should be possible to directly estimate when these satellites were accreted, exploiting the fact that disrupting satellites form separate clumps in frequency space and the separation of these clumps relate to the time since accretion (McMillan \& Binney, 2008; Gómez \& Helmi, 2010). This remarkable feat requires a large enough sample of stars with accurate parallaxes (typically 50 or more stars with parallax error less than 2 per cent), but in this realization Gómez et al. predict that it should be attainable for at least four of their detected satellites. Being able to determine the time of accretion, together with a detailed analysis of the chemistry of these stars, will undoubtedly teach us a great deal about the evolution of star formation in these earliest galaxies.

With Gaia in mind, a number of other studies have devised methods to search for substructures. One such work is that of Mateu et al. (2011), who utilise the fact that (for a spherical potential) streams will fall on great circles as viewed from the Galactic centre. This is based on an earlier study (Johnston et al., 1996), but by extending the analysis to include data such that will be available from Gaia (i.e. parallaxes and kinematics) the method has much greater efficacy. Of course this technique still requires streams to be confined to orbital planes and, as such, is illsuited to the inner halo where the shorter dynamical times lead to significant phase mixing. However, at intermediate distances in the halo where Gaia will still be able to provide reasonable parallaxes (with distance accuracy of say 30 per cent), this technique will thrive.

Although Gaia will play a dominant role in the coming decades, it will not provide all of the answers. The on-board spectroscopy is limited to only the brightest stars and will not deliver detailed chemistry, meaning that a huge ground-based follow-up program is required. The realization that this limitation hampered the scientific return of the Hipparcos mission, led to the Gaia-ESO survey, and also provides strong motivation for future instruments, such as Subaru's Prime Focus Spectrograph (Takada et al., 2014), 4-MOST (de Jong et al., 2014), WEAVE (Dalton et al., 2014) and the Maunakea Spectroscopic Explorer (Simons et al., 2014).

It is fascinating to see how, 150 years since Mädler and his contemporaries made their first discoveries, the analysis of kinematic substructures is still playing an important role in understanding the evolution of the Galaxy. Mädler couldn't have imagined that some moving groups could be the relics of other galaxies, but to- 


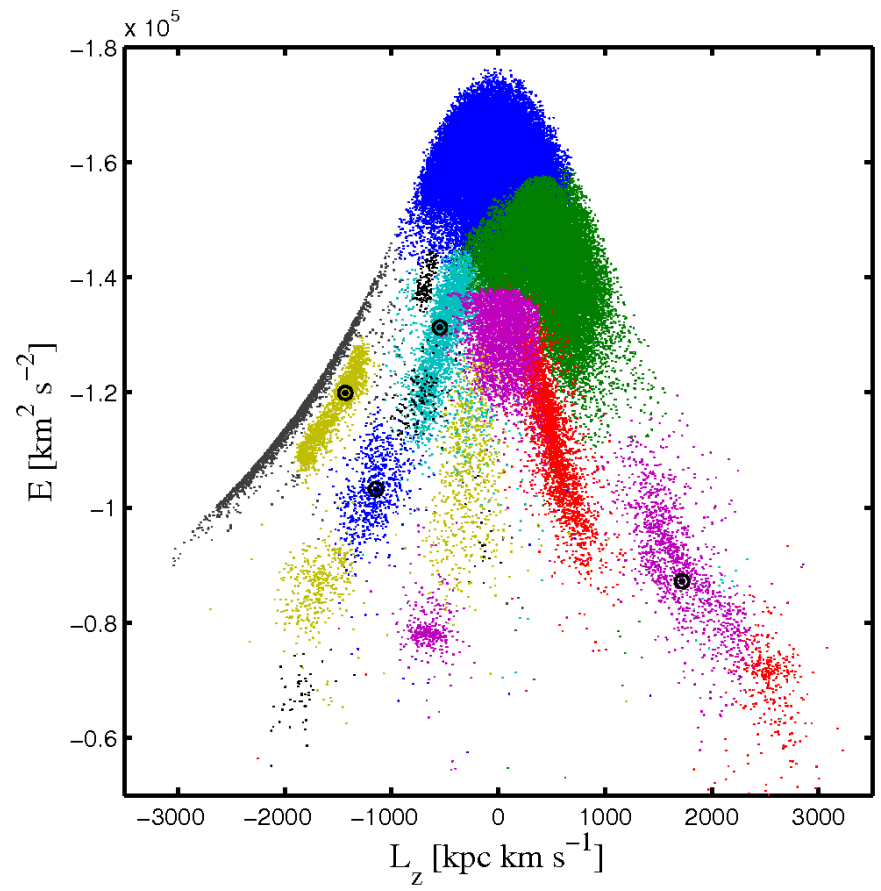

Fig. 10 An example of how Gaia might see the distribution of accreted satellites in the solar neighbourhood. The different colours correspond to different satellites in the space of energy and the vertical component of the angular momentum. Black circles denote the four satellites for which it will be possible to estimate the time of accretion. Taken from Gómez et al. (2010).

day these substructures are illuminating our knowledge of the earliest galaxies and hierarchical assembly. As new surveys are undertaken, the census of substructures becomes more complete. Perhaps in 150 years accretion events such as these will still be contributing new insights.

Acknowledgements The author acknowledges financial support from the CAS One Hundred Talent Fund, NSFC grants 11173002 and 11333003, the National Key Basic Research Program of China (2014CB845700) and the Strategic Priority Research Program "The Emergence of Cosmological Structures" of the Chinese Academy of Sciences (XDB09000000). This work is partially supported by the Gaia Research for European Astronomy Training (GREAT-ITN) Marie Curie network, funded through the European Union Seventh Framework Programme (FP7/2007-2013) under grant agreement No. 264895. This chapter uses data obtained through the Telescope Access Program (TAP), which has been funded by the Strategic Priority Research Program "The Emergence of Cosmological Structures" (Grant No. XDB09000000), National Astronomical Observatories, Chinese Academy of Sciences, and the Special Fund for Astronomy from the Ministry of Finance. 


\section{References}

Antoja, T., Figueras, F., Torra, J., Valenzuela, O., \& Pichardo, B. 2010, Lecture Notes and Essays in Astrophysics, 4, 13

Arifyanto, M. I., \& Fuchs, B. 2006, A\&A, 449, 533

Beers, T. C., Chiba, M., Yoshii, Y., et al. 2000, AJ, 119, 2866

Beers, T. C., \& Sommer-Larsen, J. 1995, ApJS, 96, 175

Belokurov, V. 2013, NewAR, 57, 100

Bensby, T., Feltzing, S., \& Oey, M. S. 2014, A\&A, 562, A71

Binney, J., \& Tremaine, S. 2008, Galactic Dynamics: Second Edition (Princeton University Press)

Binney, J., Burnett, B., Kordopatis, G., et al. 2014a, MNRAS, 439, 1231

-. 2014b, MNRAS, 437, 351

Bobylev, V. V., Bajkova, A. T., \& Mylläri, A. A. 2010, Astronomy Letters, 36, 27

Bochanski, J. J., Savcheva, A., West, A. A., \& Hawley, S. L. 2013, AJ, 145, 40

Boeche, C., Siebert, A., Williams, M., et al. 2011, AJ, 142, 193

Bovy, J., \& Hogg, D. W. 2010, ApJ, 717, 617

Bovy, J., Hogg, D. W., \& Roweis, S. T. 2009, ApJ, 700, 1794

Bovy, J., Rix, H.-W., \& Hogg, D. W. 2012, ApJ, 751, 131

Bovy Jo, Hogg, D. W., \& Roweis, S. T. 2011, Annals of Applied Statistics, 5, 1657

Bramich, D. M., Vidrih, S., Wyrzykowski, L., et al. 2008, MNRAS, 386, 887

Breddels, M. A., Smith, M. C., Helmi, A., et al. 2010, A\&A, 511, A90

Carney, B. W., Latham, D. W., Laird, J. B., \& Aguilar, L. A. 1994, AJ, 107, 2240

Casagrande, L., Schönrich, R., Asplund, M., et al. 2011, A\&A, 530, A138

Casey, A. R., Keller, S. C., Alves-Brito, A., et al. 2014, MNRAS, 443, 828

Chiba, M., \& Beers, T. C. 2000, AJ, 119, 2843

Chiba, M., \& Yoshii, Y. 1998, AJ, 115, 168

Clewley, L., \& Kinman, T. D. 2006, MNRAS, 371, L11

Cooper, A. P., Cole, S., Frenk, C. S., \& Helmi, A. 2011, MNRAS, 417, 2206

Dalton, G., Trager, S., Abrams, D. C., et al. 2014, in Society of Photo-Optical Instrumentation Engineers (SPIE) Conference Series, Vol. 9147, Society of PhotoOptical Instrumentation Engineers (SPIE) Conference Series, 0

de Boer, T. J. L., Belokurov, V., Beers, T. C., \& Lee, Y. S. 2014, MNRAS, 443, 658 de Bruijne, J. H. J. 2012, Ap\&SS, 341, 31

de Jong, R. S., Barden, S., Bellido-Tirado, O., et al. 2014, in Society of PhotoOptical Instrumentation Engineers (SPIE) Conference Series, Vol. 9147, Society of Photo-Optical Instrumentation Engineers (SPIE) Conference Series, 0

Dehnen, W., \& Binney, J. J. 1998, MNRAS, 298, 387

Dekker, E. 1976, Phys. Rep., 24, 315

Deng, L.-C., Newberg, H. J., Liu, C., et al. 2012, Research in Astronomy and Astrophysics, 12, 735

Dettbarn, C., Fuchs, B., Flynn, C., \& Williams, M. 2007, A\&A, 474, 857

Dinescu, D. I., Girard, T. M., \& van Altena, W. F. 1999, AJ, 117, 1792

Eggen, O. J. 1978, ApJ, 221, 881

-.1996, AJ, 112, 1595 
ESA, ed. 1997, ESA Special Publication, Vol. 1200, The HIPPARCOS and TYCHO catalogues. Astrometric and photometric star catalogues derived from the ESA HIPPARCOS Space Astrometry Mission

Famaey, B., Siebert, A., \& Jorissen, A. 2008, A\&A, 483, 453

Frebel, A., Lunnan, R., Casey, A. R., et al. 2013, ApJ, 771, 39

Freeman, K., \& Bland-Hawthorn, J. 2002, ARA\&A, 40, 487

Freeman, K. C. 2012, in Astronomical Society of the Pacific Conference Series, Vol. 458, Galactic Archaeology: Near-Field Cosmology and the Formation of the Milky Way, ed. W. Aoki, M. Ishigaki, T. Suda, T. Tsujimoto, \& N. Arimoto, 393

Fuchs, B., \& Dettbarn, C. 2011, AJ, 141, 5

Gilmore, G., Randich, S., Asplund, M., et al. 2012, The Messenger, 147, 25

Gómez, F. A., \& Helmi, A. 2010, MNRAS, 401, 2285

Gómez, F. A., Helmi, A., Brown, A. G. A., \& Li, Y.-S. 2010, MNRAS, 408, 935

Gould, A. 2003, ApJ, 592, L63

Harrigan, M. J., Newberg, H. J., Newberg, L. A., et al. 2010, MNRAS, 405, 1796

Helmi, A. 2008, A\&A Rev., 15, 145

Helmi, A., Navarro, J. F., Nordström, B., et al. 2006, MNRAS, 365, 1309

Helmi, A., \& White, S. D. M. 1999, MNRAS, 307, 495

Helmi, A., White, S. D. M., de Zeeuw, P. T., \& Zhao, H. 1999, Nature, 402, 53

Helmi, A., Williams, M., Freeman, K. C., Bland-Hawthorn, J., \& De Silva, G. 2014, ApJ, 791, 135

Holmberg, J., Nordström, B., \& Andersen, J. 2007, A\&A, 475, 519

-. 2009, A\&A, 501, 941

Holtzman, J. A., Shetrone, M., Johnson, J. A., et al. 2015, ArXiv e-prints, arXiv: 1501.04110

Ibata, R. A., Gilmore, G., \& Irwin, M. J. 1994, Nature, 370, 194

Ivezić, Ž., Beers, T. C., \& Jurić, M. 2012, ARA\&A, 50, 251

Ivezić, Ž., Sesar, B., Jurić, M., et al. 2008, ApJ, 684, 287

Johnston, K. V., Bullock, J. S., Sharma, S., et al. 2008, ApJ, 689, 936

Johnston, K. V., Hernquist, L., \& Bolte, M. 1996, ApJ, 465, 278

Jurić, M., Ivezić, Ž., Brooks, A., et al. 2008, ApJ, 673, 864

Kapteyn, J. C., ed. 1906, Plan of Selected Areas, Astronomical Laboratory at Groningen

Kepley, A. A., Morrison, H. L., Helmi, A., et al. 2007, AJ, 134, 1579

Kinman, T. D. 2000, in Astrophysics and Space Science Library, Vol. 246, Astrophysics and Space Science Library, ed. P. C. van der Kruit \& K. van Berkel, 213-228

Klement, R., Fuchs, B., \& Rix, H.-W. 2008, ApJ, 685, 261

Klement, R., Rix, H.-W., Flynn, C., et al. 2009, ApJ, 698, 865

Klement, R. J. 2010, A\&A Rev., 18, 567

Klement, R. J., Bailer-Jones, C. A. L., Fuchs, B., Rix, H.-W., \& Smith, K. W. 2011, ApJ, 726, 103

Koposov, S. E., Belokurov, V., \& Wyn Evans, N. 2013, ApJ, 766, 79

Koposov, S. E., Rix, H.-W., \& Hogg, D. W. 2010, ApJ, 712, 260

Kordopatis, G., Gilmore, G., Steinmetz, M., et al. 2013, AJ, 146, 134 
Lee, D. M., Johnston, K. V., Sen, B., \& Jessop, W. 2015, ApJ, 802, 48

Lee, Y. S., Beers, T. C., Sivarani, T., et al. 2008, AJ, 136, 2022

Lee, Y. S., Beers, T. C., Allende Prieto, C., et al. 2011, AJ, 141, 90

Lee, Y.-W., Joo, J.-M., Sohn, Y.-J., et al. 1999, Nature, 402, 55

Mädler, J. H. 1846, Astronomische Nachrichten, 24, 213

Majewski, S. R., Nidever, D. L., Smith, V. V., et al. 2012, ApJ, 747, L37

Mateu, C., Bruzual, G., Aguilar, L., et al. 2011, MNRAS, 415, 214

McMillan, P. J. 2011, MNRAS, 418, 1565

McMillan, P. J., \& Binney, J. J. 2008, MNRAS, 390, 429

Navarro, J. F., Helmi, A., \& Freeman, K. C. 2004, ApJ, 601, L43

Newberg, H. J., Yanny, B., \& Willett, B. A. 2009, ApJ, 700, L61

Nordström, B., Mayor, M., Andersen, J., et al. 2004, A\&A, 418, 989

Proctor, R. A. 1869, Royal Society of London Proceedings Series I, 18, 169

Ramya, P., Reddy, B. E., \& Lambert, D. L. 2012, MNRAS, 425, 3188

Randich, S., Gilmore, G., \& Gaia-ESO Consortium. 2013, The Messenger, 154, 47

Re Fiorentin, P., Helmi, A., Lattanzi, M. G., \& Spagna, A. 2005, A\&A, 439, 551

Roederer, I. U., Sneden, C., Thompson, I. B., Preston, G. W., \& Shectman, S. A. 2010, ApJ, 711, 573

Schlaufman, K. C., Rockosi, C. M., Lee, Y. S., Beers, T. C., \& Allende Prieto, C. 2011, ApJ, 734, 49

Schlaufman, K. C., Rockosi, C. M., Lee, Y. S., et al. 2012, ApJ, 749, 77

Schlaufman, K. C., Rockosi, C. M., Allende Prieto, C., et al. 2009, ApJ, 703, 2177

Seabroke, G. M., Gilmore, G., Siebert, A., et al. 2008, MNRAS, 384, 11

Sellwood, J. A. 2010, MNRAS, 409, 145

Simons, D. A., Crampton, D., Côté, P., et al. 2014, in Society of Photo-Optical Instrumentation Engineers (SPIE) Conference Series, Vol. 9145, Society of PhotoOptical Instrumentation Engineers (SPIE) Conference Series, 15

Smith, M. C., Okamoto, S., Yuan, H.-B., \& Liu, X.-W. 2012, Research in Astronomy and Astrophysics, 12, 1021

Smith, M. C., Wyn Evans, N., \& An, J. H. 2009a, ApJ, 698, 1110

Smith, M. C., Evans, N. W., Belokurov, V., et al. 2009b, MNRAS, 399, 1223

Starkenburg, E., Helmi, A., Morrison, H. L., et al. 2009, ApJ, 698, 567

Steinmetz, M., Zwitter, T., Siebert, A., et al. 2006, AJ, 132, 1645

Stonkutė, E., Tautvaišienė, G., Nordström, B., \& Ženovienė, R. 2012, A\&A, 541, A157

-. 2013, A\&A, 555, A6

Takada, M., Ellis, R. S., Chiba, M., et al. 2014, PASJ, 66, 1

Ženovienė, R., Tautvaišienè, G., Nordström, B., \& Stonkutè, E. 2014, A\&A, 563, A53

van der Kruit, P. C., ed. 2015, Astrophysics and Space Science Library, Vol. 416, Jacobus Cornelius Kapteyn

van Leeuwen, F., ed. 2007a, Astrophysics and Space Science Library, Vol. 350,

Hipparcos, the New Reduction of the Raw Data

van Leeuwen, F. 2007b, A\&A, 474, 653 
Williams, M. E. K., Freeman, K. C., Helmi, A., \& RAVE Collaboration. 2009, in IAU Symposium, Vol. 254, IAU Symposium, ed. J. Andersen, Nordströara, B. m, \& J. Bland-Hawthorn, 139-144

Williams, M. E. K., Steinmetz, M., Sharma, S., et al. 2011, ApJ, 728, 102

Wylie-de Boer, E., Freeman, K., \& Williams, M. 2010, AJ, 139, 636

Wylie-de Boer, E., Freeman, K., Williams, M., et al. 2012, ApJ, 755, 35

Xia, Q., Liu, C., Xu, Y., et al. 2015, MNRAS, 447, 2367

Xue, X.-X., Rix, H.-W., Yanny, B., et al. 2011, ApJ, 738, 79

Yanny, B., Rockosi, C., Newberg, H. J., et al. 2009, AJ, 137, 4377

York, D. G., Adelman, J., Anderson, Jr., J. E., et al. 2000, AJ, 120, 1579

Zhao, J. K., Zhao, G., Chen, Y. Q., et al. 2014, ApJ, 787, 31

Zwitter, T., Matijevič, G., Breddels, M. A., et al. 2010, A\&A, 522, A54 